\title{
Sulla teoria dei ponti sospesi e l'impiego delle funzioni d'influenza.
}

\author{
Memoria di Pedro B. J. Gravina (a São Paulo).
}

A Mauro Picone nel suo $70^{\text {mo }}$ compleanno.

\begin{abstract}
Sunto. - In questa memoria è trattato il calcolo dei ponti sospesi secondo la Teoria del secondo ordine e mediante l'impiego delle funzioni d'influenza ristretta per gli sforzi, spostamenti e rotazioni. Vengono dedotte le espressioni di tali funzioni attraverso l'integraziore diretta delle equazioni fondamentali della Teoria e ne è dato anche lo suiluppo in serie trigonometriche.
\end{abstract}

\section{Introduzione.}

Lo studio della Teoria dei ponti sospesi, iniziatasi con i lavori di NA. vikr [1] nel 1823, si è sviluppato attraverso numerosi contributi di diversi Autori.

Dalle prime ricerche sui fili isolati si è passato alla «Teoria Elastica» di Cadart [3] e di M. Levy [4], e finalmente alla "genauere Theorie» di Müller-Breslad [2] e di J. Melan [5], chiamata anche "Deflection Theory » dagli Autori americani. È, quest'ultima teoria, quella che si applica nello studio dei grandi ponti sospesi moderni. Essa tiene conto, contrariamente a ciò che succede con la Teoria Elastica, della deformazione del sistema sotto l'azione dei carichi accidentali, ed è perciò una teoria del secondo ordine.

In questa memoria studiamo il caso dei ponti sospesi con cavi ancorati esternamente alle estremità e travi irrigidenti semplicemente appoggiate, soggette a carichi considerati agenti staticamente. Indicate le equazioni fondamentali della teoria del secondo ordine e risoltele direttamente, ricaviamo le funzioni d'influenza degli sforzi, spostamenti e rotazioni, di comodo impiego per le applicazioni. Ricaviamo, inoltre, le espressioni ottenute nello studio precedente, sviluppate in serie trigonometriche che, in alcuni casi, sono di impiego comodo per la rapida convergenza che le caratterizza.

\section{Equazioni fondamentali della teoria.}

Le ipotesi fatte dalla teoria del secondo ordine dei ponti sospesi, sono, in genere, le seguenti :

1 - Le tensioni nei diversi elementi della struttura non superano il limite di proporzionalita del materiale impiegato. 
2 - Il cavo sostiene il peso proprio $w$ del ponte rimanendo, pertanto, la trave irrigidente priva di sforzi quando non agiscono le forze accidentali sul sistema.

3 - La configurazione iniziale di equilibrio dell' asse del cavo sotto l'azione del peso proprio $w$ è una parabola.

4 - Il cavo è perfettamente flessibile.

5 - La distanza tra $\mathrm{i}$ tiranti di sospensione è molto piccola in relazione alla distanza tra gli appoggi della trave irrigidente.

6 - Sono trascurabili gli effetti dovati all'allungamento dei tiranti di sospensione e all'accorciamento delle torri sotto l'azione del carico mobile.

7 - I carrelli di appoggio del cavo sulle torri non soffrono impedimento di alcun genere agli spostamenti orizzontali.

8 - Sono trascurabili le deformazioni della trave irrigidente dovute allo sforzo di taglio e allo sforzo normale.

9 - Il momento d'inerzia della trave irrigidente è costante lungo ognuno dei vani del ponte.

Considerando un tratto $d x$ del ponte (trave irrigidente e cavo) sotto l'azione dei carichi permanente e accidentali e scrivendo le corrispondenti condizioni statiche di equilibrio, si perviene facilmente [15] all'equazione di Müliter Bresinau-Melan :

$$
p(x)=E J \eta^{\mathrm{IV}}-H_{S} y^{\prime \prime}-H \eta^{\prime \prime}
$$

dove $p(x)$ è il carico mobile per unità di lunghezza; $E$ il modulo di elasticità e $J$ il momento d'inerzia della trave irrigidente; $\eta$ lo spostamento verticale della sezione del cavo e della trave con la stessa ascissa $x$, provocato dalle forze accidentali; $H_{S}$ la spinta addizionale dovuta alle forze accidentali; $y$ l'ordinata dell'asse del cavo; $H$ la spinta totale dovuta al carico permanente e alle forze accidentali.

Per trasformare l'equazione (1) in una relazione con termini adimensionali, moltiplichiamo il primo e il secondo membro per $l^{3} / E J$, dove $l$ è una lunghezza fissa scelta arbitrariamente. Abbiamo allora:

$$
l^{3} \eta^{\mathrm{Iv}}-c^{2} l \eta^{\prime \prime}=\frac{c^{2}}{1+\beta}\left(\beta l y^{\prime \prime}+\frac{p(x) l}{H_{w}}\right)
$$

dove :

$$
\beta=\frac{H_{S}}{H_{w}}
$$

essendo $H_{w}$ la spinta dovuta al carico permanente $w$. e

$$
c^{2}=\frac{H l^{2}}{E J}=\frac{(1+\beta) H_{w} l^{2}}{E J} .
$$




\section{Chiameremo:}

$$
c_{0}=\sqrt{\frac{\overline{H_{w} l^{2}}}{E J}}
$$

di indice di flessibilità propria del ponte nel vano generico e

$$
c=c_{0} \sqrt{1+\beta}
$$

di indice di flessibilita accidentale del ponte nel vano generico.

Essendo :

$$
H=H_{S}+H_{w}
$$

l'equazione (1) fornisce una relazione tra lo spostamento $\eta$ e la spinta $H$ che sono le due incognite del problema.

Occorre, pertanto, un'altra relazione che ei è fornita dall'equazione di condizione del cavo. Questa equazione si ottiene esprimendo le condizioni di deformazione del cavo e osservando che la sua proiezione orizzontale, dopo l'azione delle forze accidentali, deve essere uguale alla distanza orizzontale tra gli ancoraggi di estremità meno l'eventuale differenza $\Delta L$ dovuta ad un cedimento dei blocchi [15]. Otteniamo cosi, con termini adimensionali, l'equazione :

$$
-\int_{C} y^{\prime \prime} \eta d \frac{x}{l}=\beta 9 \cdot \frac{L_{S}}{l}+\omega t \frac{L}{l}-\frac{\Delta L}{l}+\frac{1}{2} \int_{C} \eta^{\prime \prime} \eta d \frac{x}{l}
$$

dove $L_{S}, L, \vartheta$ sono delle costanti che dipendono dalle caratteristiche del cavo e dalla sua configurazione iniziale di equilibrio:

$$
\begin{gathered}
L_{S}=\int_{C} \sec ^{3} \varphi d x \\
L=\int_{c} d x \\
\vartheta=\frac{H_{w}}{E_{C} A_{C}}
\end{gathered}
$$

$\omega$ a il coefficiente di dilatazione termica del eavo, $t$ la variazione uniforme di temperatura, $E_{C}$ il modulo di elasticità e $A_{C}$ l'area della sezione del cavo, $\varphi$ l'angolo generico che la tangente all' asse del cavo, soggetto al carico permanente, forma con l'orizzontale. L'indice $C$ presso il segno d'integrale vaol significare che l'integrazione si stende a tutta la lunghezza del cavo.

In genere si suole trascurare nelle applicazioni l'ultimo termine del secondo membro dell'equazione (8) con il quale si tiene conto della variazione, lungo il vano della frazione di carico mobile ohe si trasmette ai tiranti di 
sospensione. L'equazione di condizione del cavo si riduce, pertanto, alla seguente:

$$
-\int_{c} y^{\prime \prime} \eta d \frac{x}{l}=\beta \vartheta \frac{L_{S}}{l}+\omega t \frac{L}{l}-\frac{\Delta L}{l}
$$

\section{Risoluzione delle equazioni fondamentali.}

Ottenute le due equazioni (1) e (12) fra le due incognite $\eta$ e $H$, possiamo risolvere il problema. Per procedere al calcolo consideriamo, in un primo tempo, costanti la variabile $H$ e pertanto $H_{S}$ dell'equazione (1). Abbiamo cosi un'equazione differenziale ordinaria, di quart' ordine, lineare, non omogenea, con coefficienti costanti, che ci fornisce la legge di variazione di $\eta$ in funzione di $x$ per il valore costante di $H$ ammesso. Essendo uguali, per ipotesi, gli spostamenti verticali delle sezioni della trave di irrigidimento e del cavo che si corrispondono, posaiamo sostituire la espressione di $\eta$ nella equazione di condizione del cavo e ottenere un valore più approssimato di $H$ e pertanto di $H_{S}$ che ei permette di dedurre, a sua volta, una legge di variazione di $\eta$ più esatta. Procedendo cosi risolveremo, pertanto, il problema adoperando il metodo delle approssimazioni successive.

Dalla teoria della trave rettilinea si sa che:

$$
M=-E J \eta^{\prime \prime} .
$$

\section{Poniamo :}

$$
k^{2}=\frac{H}{E d}
$$

donde per la (4):

$$
c=k l \text {. }
$$

Possiamo così trasformare la (1) nella seguente:

$$
-M^{\prime \prime}+k^{2} M=H_{S} y^{\prime \prime}+p(x) .
$$

Dalla teoria dei fili sappiamo che:

$$
w(x)+H_{w} y^{\prime \prime}=0
$$

donde :

$$
-M^{t}+k^{2} M=p(x)-\beta w(x) .
$$

Per il vano $n$ è $k=k_{n}$ e $w(x)=w_{n}$, carico permanente costante del ponte nel vano $n$, per unità di Iunghezza. Donde:

$$
M^{\prime \prime}-k_{n}^{2} M=f(x)
$$


dove :

(19)

$$
f(x)=\beta w_{n}-p(x)
$$

La risoluzione dell' equazione (18) non omogenea, con le condizioni ai limiti omogenee:

$$
M(0)=0, \quad M\left(l_{n}\right)=0
$$

costituisce un problema di contorno omogeneo. La soluzione del problema ̀̀, pertanto :

$$
M(x)=-\int_{0}^{l_{n}} G_{1}(x, z) f(z) d z
$$

dove $G_{1}(x, z)$ è la funzione di Grees del problema omogeneo associato:

$$
\begin{gathered}
M^{\prime \prime}-k_{n}^{2} M=0 \\
M(0)=0, \quad M\left(l_{n}\right)=0 .
\end{gathered}
$$

Conosciute due soluzioni $M_{1}(x)$ e $M_{2}(x)$ dell' equazione differenziale (22) che soddisfano rispettivamente, alla prima e alla seconda condizione ai limiti (23), possiamo costruire facilmente la funzione di GreEN del problema omogeneo associato:

$$
\begin{gathered}
G_{1}(x, z)= \begin{cases}\frac{M_{1}(x) M_{1}(z)}{C} & \text { quando } 0 \leqq x \leqq z, \\
\frac{M_{2}(x) M_{1}(z)}{C} & \text { quando } z \leqq x \leqq l_{n} .\end{cases} \\
C=M_{2}(z) M_{1}^{\prime}(z)-M_{1}(z) M_{2}^{\prime}(z)=M_{2}(0) M_{1}^{\prime}(0)=-M_{1}\left(l_{n}\right) M_{2}^{\prime}\left(l_{n}\right) .
\end{gathered}
$$

Essendo :

$$
M_{1}(x)=\operatorname{sh} k_{n} x, \quad M_{2}(x)=\operatorname{sh} k_{n}\left(l_{n}-x\right)
$$

abbiamo:

$$
G_{1}(x, z)= \begin{cases}\frac{\operatorname{sh} k_{n} x \operatorname{sh} k_{n}\left(l_{n}-z\right)}{k_{n} \operatorname{sh} k_{n} l_{n}} & \text { quando } 0 \leqq x \leqq z, \\ \frac{\operatorname{sh~} k_{n}\left(l_{n}-x\right) \operatorname{sh} k_{n} z}{k_{n} \operatorname{sh} k_{n} l_{n}} & \text { quando } z \leqq x \leqq l_{n} .\end{cases}
$$

Osservando che nell' espressione (19) di $f(x)$ è $\beta w_{n}$ costante, abbiamo dalla (21):

$$
\begin{aligned}
& M(x)= \frac{\operatorname{sh} k_{n}\left(l_{n}-x\right) \int_{0}^{\infty} p(z) \operatorname{sh} k_{n} z d z+\operatorname{sh} k_{n} x \int_{x}^{l_{n}} p(z) \operatorname{sh} k_{n}\left(l_{n}-z\right) d z}{k_{n} \operatorname{sh} k_{n} l_{n}}+ \\
&+\beta \frac{w_{n}}{k_{n}^{2}}\left[\frac{\operatorname{ch} k_{n}\left(\frac{l_{n}}{2}-x\right)}{\operatorname{ch} \frac{k_{n} l_{n}}{2}}-1\right] .
\end{aligned}
$$


Conosciuta la funzione $M(x)$ possiamo calcolare lo spostamento $\eta(x)$ risol. vendo il problema non omogeneo:

$$
\begin{gathered}
\eta^{\prime \prime}=-\frac{M(x)}{E_{n}} \\
\eta(0)=0, \quad \eta\left(l_{n}\right)=0 .
\end{gathered}
$$

La funzione di Green del problema omogeneo associato è :

$$
G_{2}(x, t)= \begin{cases}\frac{1}{l_{n}} x\left(l_{n}-t\right) & \text { quando } 0 \leqq x \leqq t, \\ \frac{1}{l_{n}} t\left(l_{n}-x\right) & \text { quando } t \leqq x \leqq l_{n},\end{cases}
$$

donde:

$$
\eta(x)=\int_{0}^{l_{n}} G_{2}(x, t) \frac{M(t)}{E J_{n}} d t
$$

che per la (21) possiamo scrivere:

$$
\frac{\eta(x)}{l}=-\int_{0}^{l_{n}} f(z) d z \int_{0}^{l_{n}} \frac{G_{2}(x, t) G_{1}(t, z)}{l E J_{n}} d t
$$

dove $l$ è una lunghezza fissa arbitraria, per cui si è diviso il primo e il secondo membro della (32).

Calcoliamo il secondo integrale del secondo membro. Quando $0 \leqq x \leqq z$ abbiamo per la (24) e la (31):

$$
\begin{aligned}
& Y_{x z}=\int_{0}^{l_{n}} \frac{G_{2}(x, t) G_{1}(t, z)}{l E J_{n}} d t=\frac{\left(l_{n}-x\right)}{l_{n}} \frac{M_{2}(z)}{C} \int_{0}^{x} t \frac{M_{1}(t)}{E J_{n}} d t+ \\
& +\frac{x}{l l_{n}} \frac{M_{2}(z)}{C} \int_{z}^{z}\left(l_{n}-t\right) \frac{M_{1}(t)}{E J_{n}} d t+\frac{x}{l l_{n}} \frac{M_{1}(z)}{C} \int_{z}^{l_{n}}\left(l_{n}-t\right) \frac{M_{z}(t)}{E J_{n}} d t .
\end{aligned}
$$

Osserviamo che per la (18) possiamo scrivere:

$$
\frac{M_{1}(t)}{E J_{n}}=\frac{M_{1}^{\prime \prime}(t)}{H_{w}+\bar{H}_{S}}, \quad \frac{M_{2}(t)}{E J_{n}}=\frac{M_{2}^{\prime \prime}(t)}{H_{w}+H_{S}}
$$


donde sostituendo nella (34), integrando per parti e osservando che $M_{1}(0)=M_{2}\left(l_{n}\right)=0$, abbiamo:

$$
\begin{gathered}
Y_{x z}=\frac{x\left(l_{n}-z\right)}{l l_{n}} \frac{\left[M_{2}(z) M_{1}^{\prime}(z)-M_{1}(z) M_{2}^{\prime}(z)\right]}{C\left(H_{w}+H_{S}\right)}- \\
-\frac{\left(l_{n}-x\right)}{l l_{n}} \frac{M_{2}(z) M_{1}(x)}{C\left(H_{w}+H_{S}\right)}-\frac{x}{l l_{n}} \frac{M_{2}(z) M_{1}(x)}{C\left(H_{w}+H_{S}\right)}= \\
=\frac{x\left(l_{n}-z\right)}{l l_{n} H_{w}(1+\beta)}-\frac{M_{2}(z) M_{1}(x)}{l C H_{w}(1+\beta)}
\end{gathered}
$$

quando $0 \leqq x \leqq z$.

Analogamente :

$$
Y_{x z}=\frac{\left(l_{n}-x\right) z}{l l_{n} H_{w}(1+\beta)}-\frac{M_{1}(z) M_{2}(x)}{l O H_{w}(1+\beta)}
$$

quando $z \leqq x \leqq l_{n}$.

Ponendo:

$$
X=\frac{x}{l_{n}}, \quad Z=\frac{z}{l_{n}}
$$

e sostituendo nelle (35) e (36) al posto di $M_{1}, M_{2}$ e $C$ le corrispondenti espressioni (26) e (25) abbiamo:

$$
Y_{x z}=\frac{1}{H_{w}(1+\beta)} \frac{l_{n}}{l}\left[X(1-Z)-\frac{\operatorname{sh} c_{n} X \operatorname{sh} c_{n}(1-Z)}{c_{n} \operatorname{sh} c_{n}}\right]
$$

quando $0 \leqq X \leqq Z$ e:

$$
Y_{x s}=\frac{1}{H_{w}(1+\beta)} \frac{l_{n}}{l}\left[(1-X) Z-\frac{\operatorname{sh} c_{n}(1-X) \operatorname{sh} c_{n} Z}{c_{n} \operatorname{sh} c_{n}}\right]
$$

quando $Z \leqq X \leqq 1$.

Poniamo adesso:

$$
\left\{\begin{array}{c}
j_{x z}=(1-X) Z-\frac{\operatorname{sh} c_{n}(1-X) \operatorname{sh} c_{n} Z}{c_{n} \operatorname{sh} c_{n}} \text { quando } Z \leqq X, \\
j_{x z}=X(1-Z)-\frac{\operatorname{sh} c_{n} X \operatorname{sh} c_{n}(1-Z)}{c_{n} \operatorname{sh} c_{n}} \text { quando } Z \geqq X, \\
j_{x}=\frac{1}{2} X(1-X)-\frac{1}{c_{n}^{2}}+\frac{\operatorname{ch} c_{n}\left(\frac{1}{2}-X\right)}{c_{n}^{2} \operatorname{ch} \frac{c_{n}}{2}}
\end{array}\right.
$$


e ancora definiamo:

$$
\begin{gathered}
J_{x z}=\frac{l_{n}}{l} j_{x z} \\
J_{x}=\frac{w_{n}}{w_{0}}\left(\frac{l_{n}}{l}\right)^{2} j_{x}
\end{gathered}
$$

dove, come abbiamo già visto, $l$ è una lunghezza fissa arbitrariamente scelta, $w_{0}$ un valore arbitrario del earico permanente, per unità di lunghezza, opportunamente fissato;

Possiamo, pertanto, scrivere le (36) e (37):

$$
Y_{x z}=\frac{1}{H_{w}(1+\beta)} J_{x z}
$$

donde la (33):

$$
\frac{\eta(x)}{l}=-\frac{1}{H_{w}(1+\beta)} \int_{0}^{l_{n}} J_{x z} f(z) d z .
$$

Sostituendo al posto di $f(z)$ il valore dato dalla (19) abbiamo:

$$
\frac{\eta(x)}{l}=\frac{u}{1+\beta}\left[\int_{0}^{l n} J_{x z} F(z) d \frac{z}{l}-\beta J_{x}\right] .
$$

dove :

$$
\begin{gathered}
u=\frac{w_{0} l}{H_{w}} \\
\frac{p(z)}{w_{0}}=F(z) \\
J_{\infty}=\int_{0}^{l} \frac{w_{n}}{w_{0}} J_{x z} d \frac{z}{l}=\frac{w_{n}}{w_{0}}\left(\frac{l_{n}}{l}\right)^{2} J_{x} .
\end{gathered}
$$

Per applicare le equazioni del momento e dello spostamento che abbiamo dedotto è necessario conoscere il valore di $\beta$ dovuto alla condizione di carico assegnata, essendo per la (6):

$$
c_{n}=c_{0 n} \sqrt{1+\beta}
$$

dove l'indice $n$ vuol significare che studiamo il problema per il vano $n$ generico. Questo valore di $\beta$ sarà fornito dalla equazione di condizione del cavo (12):

$$
-\int y^{\prime \prime} \eta d \frac{x}{l}=\beta{ }^{9} \frac{L_{S}}{l}+\omega t \frac{L}{l}-\frac{\Delta L}{l} .
$$


Per potere calcolare l'integrale a primo membro è necessario conoscere la relazione che intercorre tra $\eta$ e $x$, rispettivamente, spostamento verticale e ascissa della sezione generica del cavo. Essendo, per ipotesi, uguali gli spostamenti verticali delle sezioni del cavo e della trave irrigidente che si corrispondono, possiamo adoperare la equazione (44) dello spostamento della trave dedotta innanzi. Abbiamo:

$$
\int_{G} \frac{u w_{n}}{w_{0}} \frac{1+\beta}{u}\left[J_{x z} F(z) d \frac{z}{l}-\beta J_{x}\right] d \frac{x}{l}=\beta \vartheta \frac{L_{S}}{l}+\omega t \frac{L}{l}-\frac{\Delta L}{l}
$$

\section{Poniamo:}

$$
A=\beta 2 \frac{L_{S}}{l}+\omega t \frac{L}{l}-\frac{\Delta L}{l}
$$

e sostituiamo al posto di $J_{x z}$ e $J_{x}$ le espressioni (40) e (41). Integrando lungo tutto il cavo abbiamo:

$$
\begin{gathered}
\frac{u^{2}}{1+\beta} \sum_{n} \frac{w_{n}}{w_{0}}\left(\frac{l_{n}}{l}\right)^{2}\left\{\int_{0}^{Z}\left[X(1-Z)-\frac{\operatorname{sh} c_{n} X \operatorname{sh} c_{n}(1-Z)}{c_{n} \operatorname{sh} c_{n}}\right] d X+\right. \\
\quad+\int_{Z}^{1}\left[(1-X) Z-\frac{\operatorname{sh} c_{n}(1-X) \operatorname{sh} c_{n} Z}{c_{n} \operatorname{sh} c_{n}}\right] d X \mid F(z) d \frac{z}{l}- \\
-\frac{u^{3} \beta}{1+\beta} \sum_{n} \frac{w_{n}}{w_{0}} \frac{w_{n}}{w_{0}}\left(\frac{l_{n}}{l}\right)^{3} \int_{0}^{1}\left[\frac{1}{2} X(1-X)-\frac{1}{c_{n}^{2}}+\frac{\operatorname{ch} c_{n}\left(\frac{1}{2}-X\right)}{c_{n}^{2} \operatorname{ch} \frac{c_{n}}{2}}\right] d X=A
\end{gathered}
$$

donde :

$$
\begin{aligned}
& \sum_{n} \frac{w_{n}}{w_{0}}\left(\frac{l_{n}}{l}\right)^{2}\left\{\frac{1}{2} Z^{2}(1-Z)-\frac{\left[\operatorname{ch} c_{n} Z-1\right] \operatorname{sh} c_{n}(1-Z)}{c_{n}^{2} \operatorname{sh} c_{n}}+\right. \\
& \left.+\frac{1}{2} Z(1-Z)^{2}-\frac{\operatorname{sh} c_{n} Z\left[\operatorname{ch~} c_{n}(1-Z)-1\right]}{c_{n}^{2} \operatorname{sh} c_{n}}\right\} F(z) d_{\frac{Z}{l}}^{Z}- \\
& \quad-\beta \sum_{n}\left(\frac{w_{n}}{w_{0}}\right)^{2}\left(\frac{l_{n}}{l}\right)^{3}\left[\frac{1}{12}-\frac{1}{c_{n}^{2}}+\frac{2 \operatorname{sh} \frac{c_{n}}{2}}{c_{n}^{3} \operatorname{ch} \frac{c_{n}}{2}}\right]=\frac{A(1+\beta)}{u^{2}}
\end{aligned}
$$


e pertanto, calcolando le espressioni racchiuse in parentesi :

$$
\begin{aligned}
& \sum_{n} \frac{w_{n}}{w_{n}}\left(\frac{l_{n}}{l}\right)^{2}\left\{\frac{1}{2} Z(1-Z)-\frac{1}{c_{n}^{2}}+\frac{\operatorname{ch} c_{n}\left(\frac{1}{2}-Z\right)}{c_{n}^{2} \operatorname{ch} \frac{c_{n}}{2}}\right\} F(z) d \frac{z}{l}- \\
& -\beta \sum_{n}\left(\frac{w_{n}}{w_{0}}\right)^{2}\left(\frac{l_{n}}{l}\right)^{3} g_{n}=\frac{A(1+\beta)}{u^{2}}
\end{aligned}
$$

dove :

$$
g_{n}=\frac{1}{12}-\frac{1}{c_{n}^{2}}+\frac{2 \operatorname{th} \frac{c_{n}}{2}}{c_{n}^{3}} .
$$

Per la (41) possiamo sorivere la (53):

$$
\sum_{n} J_{z} F(z) d_{\bar{l}}^{z}-\beta G=A \frac{(1+\beta)}{u^{2}}
$$

dove :

$$
\begin{gathered}
J_{z}=\frac{w_{n}}{w_{0}}\left(\frac{l_{n}}{l}\right)^{2} j_{z} \\
G=\sum_{n}\left(\frac{w_{n}}{w_{n}}\right)^{2}\left(\frac{l_{n}}{l}\right)^{3} g_{n}=\sum_{n} G_{n} .
\end{gathered}
$$

Se supponiamo caricato solamente il vano $n$, con la forza $F(z) d \frac{z}{l}$, pos. siamo scrivere :

$$
\frac{1}{G} J_{z} F(z) d \frac{z}{l}-\beta=\delta
$$

dove :

$$
\frac{A(1+\beta)}{u^{2} G}=\delta
$$

è il termine correttivo che tiene conto dell'elasticitì del cavo, della variazione di temperatura $\Theta$ della variazione di distanza $\Delta L$ tra gli ancoraggi.

Notiamo che in tutte queste formule che abbiamo dedotto, come in tutte le altre che dedurremo in seguito, rappresentiamo con $\beta$ il valore del rapporto $H_{S} / H_{w}$ corrispondente alla condizione di cariso che si considera.

Ponendo:

$$
\beta_{0}=\frac{1}{G} J_{z} F(z) d \frac{z}{l}
$$

possiamo serivere la (58):

$$
\beta=\beta_{0}-\delta \text {. }
$$


Caso un sistema di forze $F(z) d_{l}^{Z}$ agisse in diversi vani del ponte avremmo:

$$
\sum_{n} \frac{J_{z}}{G} F(z) d \frac{z}{l}-\beta=\delta
$$

e se le forze fossero distribuite :

$$
\sum_{n} \frac{1}{G} \int_{0}^{l_{n}} J_{z} F(z) d \frac{z}{l}-\beta=\delta .
$$

Per maggior chiarezza delle formule adopereremo l'indice $z$ nei simboli corrispondenti ai vani in eui agiscono carichi nella sezione generica di ascissa $z$.

Moltiplicando e dividendo i termini della sommatoria a primo membro della (63) per $G_{z}$ abbiamo:

$$
\sum_{n} \frac{G_{z}}{G} \beta_{0 z}-\beta=\delta
$$

dove:

$$
\beta_{0 z}=\int_{0}^{l} \frac{J_{z}}{G_{z}} F(z) d \frac{z}{l} .
$$

Per le (56) e (57) possiamo scrivere:

$$
\frac{J_{z}}{G_{z}}=\frac{\frac{w_{z}}{w_{0}}\left(\frac{l_{z}}{l}\right)^{2} j_{z}}{\left(\frac{w_{z}}{w_{0}}\right)^{2}\left(\frac{l_{z}}{l}\right) g_{z}}=\frac{w_{0}}{w_{z}} \frac{l_{z}}{l} \frac{j_{z}}{g_{z}} .
$$

Dalle (61) e (63) risulta che il valore di $\beta_{0}$ relativo ad una condizione di carico assegnata si ottiene sommando $i$ valori degli integrali :

$$
\int_{0}^{l} \frac{J_{z}}{G} F(z) d \frac{z}{l}
$$

calcolati per tutti i vani caricati del ponte. Per far ciò si traccia per ognuno dei vani caricati $l_{z}$, il diagramma della funzione $J_{z} / G$ con le ascisse $\frac{z}{l}$, supponendo $c_{z}$ costante. Calcolata l'area elementare $\frac{J_{z}}{G} d \frac{z}{l}$, si moltiplica questa per $F(z)$ e si integra lungo tutto il vano considerato. Risulta, pertanto, che il diagramma della funzione $\frac{J_{z}}{G}$ è linea $d$ 'influenza di $\beta_{0}$, supposto costante il valore di $c_{z}$. 
Per il calcolo di $\beta$ conviene adoperare la formula (64) che per la (65) possiamo scrivere:

$$
\beta=\sum_{n} \frac{G_{z}}{G} \int_{0}^{l_{z}} \frac{J_{z}}{G_{z}} F(z) d \frac{z}{l}-\delta .
$$

Il secondo membro di questa equazione è funzione di $c$ che, a sua volta, dipende da $\beta$. Il calcolo sard condotto col metodo delle approssimazioni successive. Per una certa condizione di carico si ammette il valore di. $c$ in uno dei vani, trascurando il termine correttivo $\delta$. I valori di $c$. negli altri vani risultano dalla condizione d'essere la spinta $H$ costante lungo tutto il cavo. Conosciuto un primo valore di $\beta_{0}^{\prime}$ e calcolato il termine correttivo, si ottengono valori più esatti di $c$. Da questi si deduce un valore di $\beta^{\prime}$ che serve di base per il calcolo di un altro $\beta^{\prime \prime}$. E così di seguito. La convergenza del metodo $\dot{e}$, in genere, molto rapida.

Nel caso di un carico $p$ costante, uniformemente distribuito lungo tutto il vano $l_{z}$, si deduce dall' equazione $(65)$ :

$$
\beta_{0 z}=\frac{p}{w_{z}} .
$$

Se il carico permanente $w_{z} \dot{\theta}$ costante in tutti i vani abbiamo allora :

$$
\beta=\frac{p}{w_{z}}-\delta
$$

espressione questa che fornisce il valore di $\beta$ nel caso che tutti i vani del ponte siano completamente caricati.

È interessante notare che passando al limite $c=\infty$ nelle formule dedotte, abbiamo le formule applicabili al calcolo delle funi flessibili isolate, mentre per $c=0$ otteniamo le formule relative al caso della Teoria Elastica [15].

\section{Impiego delle serie trigonometriche.}

Vogliamo, adesso, sviluppare le formule innanzi ottenute in serie trigonometriche che in alcuni casi, per la rapida convergenza che presentano, sono molto convenienti nelle applicazioni pratiche.

Le serie trigonometriche furono adoperate per la prima volta nel 1927 dal MarTin [6] e indipendentemente nel 1928 dal Trmoshenko [7] come

mezzo semplice ed éfficace per il calcolo dei ponti sospesi. Questi Autori adoperarono direttamente le serie trigonometriche per risolvere l'equazione (1) di Müller Brestiau-Melan. 
Se un carico $P$ isolato agisce in un certo vano di un ponte, il valore di $\eta / l$ in una sezione di ascissa $X$ si ottiene dalla (44) sostituendo $P$ al posto di $p(z) d z$ e sopprimendo il segno d'integrale:

$$
\frac{\eta}{l}=\frac{w_{0} l}{(1+\beta) H_{w}}\left[J_{x z} \frac{P}{w_{0} l}-\beta J_{x}\right] \text {. }
$$

Essendo $\frac{\eta}{l}=f(X)$ una funzione finita e continua nell' intervallo $0 \leqq X \leqq 1$, che si ánnulla all' estremità di questo, la possiamo sviluppare in serie trigonometrica nell' intervallo considerato.

Supposta la funzione $f(X)$ definita nell'intervallo $0 \leqq X \leqq 2$, poniamo $\pi X=t$. Abbiamo, pertanto, $f(X)=F(t)$ e l'intervallo di definizione sarà $0 \leqq t \leqq 2 \pi$. Siccome ci interessa di conoscere lo sviluppo di $f(X)=F(t)$ in serie trigonometrica per adoperarlo soltanto nell' intervallo $0 \leqq X \leqq 1$, cioè a dire $0 \leqq t \leqq \pi$, poniamo per $X<1, f(2-X)=-f(X)$, cîoè a dire $F(2 \pi-t)=$ $=-F(t)$, donde otteniamo lo sviluppo in seni :

$$
f(X)=\sum_{m=1}^{\infty} a_{m} \operatorname{sen} m \pi X
$$

essendo :

$$
a_{m}=\frac{1}{2} \int_{0}^{1} f(X) \text { sen } m \pi X d X
$$

Ponendo :

$$
\begin{gathered}
A=\frac{w_{0} l}{(1+\beta) H_{w}} \frac{l_{n}}{l} \frac{P}{w_{0} l} \\
B=\frac{\beta}{1+\beta} \frac{w_{0} l}{H_{w}} \frac{w_{n}}{w_{0}}\left(\frac{l_{n}}{l}\right)^{2}
\end{gathered}
$$

otteniamo :

$$
f(X)=A j_{x z}-B j_{x}=\sum_{m=1}^{\infty} a_{m} \text { sen } m \pi X
$$

donde :

$$
a_{m}=2 A \int_{0}^{1} j_{x z} \operatorname{sen} m \pi X d X-2 B \int_{0}^{1} j_{x} \operatorname{sen} m \pi X d X .
$$

Sostituendo in questa espressione $\mathrm{i}$ valori di $j_{x z}$ e $j_{x}$ forniti dalle (38) e (39), e calcolando gli integrali risulta :

$$
a_{m}=\frac{2 P}{(1+\beta) H_{w}} \frac{l_{n}}{l} \frac{c_{n}^{2} \operatorname{sen} m \pi Z}{m^{2} \pi^{2}\left(c_{n}^{2}+m^{2} \pi^{2}\right)}-\frac{2 \beta}{1+\beta} \frac{l w_{n}}{H_{w}}\left(\frac{l_{n}}{l}\right)^{2} \frac{c_{n}^{2}(1-\cos m \pi)}{m^{3} \pi^{3}\left(c_{n}^{2}+m^{2} \pi^{2}\right)}
$$


donde per la (75):

$$
\begin{gathered}
\frac{\eta}{l}=\frac{2 P c_{n}^{2}}{(1+\beta) H_{w} \pi^{2}} \frac{l_{n}}{l} \sum_{m=1,2,3, \ldots}^{\infty} \frac{\operatorname{sen} m \pi Z \operatorname{sen} m \pi X}{m^{2}\left(c_{n}^{2}+m^{2} \pi^{2}\right)}- \\
-\frac{4 \beta}{(1+\beta)} \frac{w_{n} l}{H_{w}} \frac{c_{n}^{2}}{\pi^{3}}\left(\frac{l_{n}}{l}\right)^{2} \sum_{m=1,3,5, \ldots}^{\infty} \frac{\operatorname{sen} m \pi X}{m^{3}\left(c_{n}^{2}+m^{2} \pi^{2}\right)} .
\end{gathered}
$$

Nel caso di carico $p$ distribuito tra due sezioni $Z_{1}$ e $Z_{2}\left(Z_{1}<Z_{2}\right)$, sostituiamo $l_{n} p d Z$ al posto di $P$ e integriamo:

$$
\begin{gathered}
\frac{\eta}{l}=\frac{2 p c_{n}^{2} l}{(1+\beta) H_{w} \pi^{3}} \frac{l_{n}^{2}}{l} \sum_{m=1,2,3, \ldots}^{\infty} \frac{\left(\cos m \pi Z_{1}-\cos m \pi Z_{2}\right) \operatorname{sen} m \pi X}{m^{3}\left(c_{n}^{2}+m^{2} \pi^{2}\right)}- \\
-\frac{4 \beta}{(1+\beta)} \frac{w_{n} l}{H_{w}} \frac{c_{n}^{2}}{\pi^{3}}\left(\frac{l_{n}}{l}\right)^{2} \sum_{m=1,3,5, \ldots}^{\infty} \frac{\operatorname{sen} m \pi X}{m^{3}\left(c_{n}^{2}+m^{2} \pi^{2}\right)} .
\end{gathered}
$$

È interessante notare che la convergenza della serie, sia nel caso della (78) come della (79), è molto rapida, bastando pochi termini per ottenere risultati ottimamente approssimati.

Le equazioni (78) e (79) si possono scrivere:

$$
\begin{gathered}
\frac{\eta}{l}=\frac{u}{1+\beta}\left[\frac{P}{w_{0} l} 2 \frac{l_{n}}{l} \sum_{m=1}^{\infty} \frac{\operatorname{sen} m \pi Z \operatorname{sen} m \pi X}{m^{2} \pi^{2} c_{n m}}-\right. \\
\left.-2 \beta \frac{w_{n}}{w_{0}}\left(\frac{l_{n}}{l}\right)^{2} \sum_{m=1}^{\infty} \frac{(1-\cos m \pi) \operatorname{sen} m \pi X}{m^{3} \pi^{3} c_{n m}}\right] \\
\frac{\eta}{l}=\frac{u}{1+\beta}\left[\frac{p}{w_{0}} 2 \frac{l_{n}^{2}}{l^{2}} \sum_{m=1}^{\infty} \frac{\left(\cos m \pi Z_{1}-\cos m \pi Z_{2}\right) \operatorname{sen} m \pi X}{m^{3} \pi^{3} c_{m m}}-\right. \\
\left.-2 \beta \frac{w_{n}}{w_{0}}\left(\frac{l_{n}}{l}\right)^{2} \sum_{m=1}^{\infty} \frac{(1-\cos m \pi) \operatorname{sen} m \pi X}{m^{3} \pi^{3} c_{n m}}\right]
\end{gathered}
$$

dove :

$$
c_{n m}=1+\frac{m^{2} \pi^{2}}{c_{n}^{2}}
$$

e $u$ è definito dalla (45). Confrontando la (80) con la (70) risulta :

$$
\begin{gathered}
J_{x z}=2 \frac{l_{n}}{l} \sum_{m=1}^{\infty} \frac{\operatorname{sen} m \pi Z \operatorname{sen} m \pi X}{m^{2} \pi^{2} c_{n m}} \\
J_{x}=2 \frac{w_{n}}{w_{0}}\left(\frac{l_{n}}{l}\right)^{2} \sum_{m=1}^{\infty} \frac{(1-\cos m \pi) \operatorname{sen} m \pi X}{m^{3} \pi^{3} c_{n m}}
\end{gathered}
$$


donde, per la (40) e per la (41):

$$
\begin{gathered}
j_{x z}=2 \sum_{m=1}^{\infty} \frac{\operatorname{sen} m \pi Z \operatorname{sen} m \pi X}{m^{3} \pi^{2} c_{n n}} \\
j_{x}=2 \sum_{m=1}^{\infty} \frac{(1-\cos m \pi) \operatorname{sen} m \pi X}{m^{3} \pi^{3} c_{n m}} .
\end{gathered}
$$

Poniamo adesso nella (71):

$$
a_{m}=\frac{u}{1+\beta} \alpha_{m}
$$

donde :

$$
\frac{\eta}{l}=\frac{u}{1+\beta} \sum_{m=1}^{\infty} \alpha_{m} \text { sen } m \pi X
$$

Risulta dalla (80), nel caso di carico $F(z) d z$ :

$$
\alpha_{m}=2 F(z) d \frac{z}{l} \cdot \frac{l_{n}}{l} \frac{\operatorname{sen} m \pi Z}{m^{2} \pi^{2} c_{n m}}-2 \beta \frac{w_{n}}{w_{0}} \frac{l_{n}^{2}}{l^{2}} \frac{1-\cos m \pi}{m^{3} \pi^{3} c_{n m}}
$$

e dalla (81) nel caso di un carico $F=\frac{p}{w_{0}}=$ costante, distribuito tra due sezioni $Z_{1}$ e $Z_{2}\left(Z_{1}<Z_{2}\right)$ :

$$
\alpha_{m}=2 F \frac{l_{n}^{2}}{l^{2}} \frac{\cos m \pi Z_{1}-\cos m \pi Z}{m^{3} \pi^{3} c_{n m}}-2 \beta \frac{w_{n}}{w_{0}} \frac{l_{n}^{2}}{l^{2}} \frac{1-\cos m \pi}{m^{3} \pi^{3} c_{n m}} .
$$

Consideriamo, adesso, l'equazione di condizione del cavo nella forma più generale (8), in cui si conserva l'ultimo termine a secondo membro che tiene conto della variazione, lungo il vano, della frazione di carico mobile trasmesso ai tiranti di sospensione. Osservando che:

$$
\eta^{\prime \prime}=\frac{d^{2} \eta}{d x^{2}}=\frac{d^{2} \eta}{l_{n}^{2} d X^{2}}
$$

otteniamo :

$$
\frac{u}{w_{0} l} \sum_{n} w_{n} l_{n} \int_{0}^{1} \frac{\eta}{l} d X=\beta \vartheta \frac{L_{S}}{l}+\omega t \frac{L}{l}-\frac{\Delta L}{l}+\frac{l}{2} \sum_{n} \frac{l_{n}}{l_{n}^{2}} \int_{0}^{1} \frac{\eta^{\prime \prime}}{l} \frac{\eta}{l} d X .
$$

Dalla (88) risulta

$$
\begin{aligned}
& \int_{0}^{1} \frac{\eta}{l} d X=\frac{u}{1+\beta} \sum_{m=1}^{\infty}\left[\frac{\alpha_{m}}{m \pi}(1-\cos m \pi)\right] \\
& \int_{0}^{1} \frac{\eta^{\prime \prime}}{l} \frac{\eta}{l} d X=-\frac{u^{2}}{(1+\beta)^{2}} \sum_{m=1}^{\infty} \alpha_{m}^{2} \frac{m^{2} \pi^{2}}{2}
\end{aligned}
$$


donde per la (91):

$$
\begin{gathered}
\beta{ }^{2} \cdot \frac{L_{S}}{l}=\frac{u^{2}}{(1+\beta) \pi w_{0} l} \sum_{n} w_{n} l_{n} \sum_{m=1}^{\infty} \frac{\alpha_{m}}{m}(1-\cos m \pi)- \\
-\omega t \frac{L}{l}+\frac{\Delta L}{l}+\frac{u^{2}}{(1+\beta)^{2}} \frac{\pi^{2} l}{4} \sum_{n} \frac{l_{n}}{l_{m}^{2}} \sum_{m=1}^{\infty} m^{2} \alpha_{m} .
\end{gathered}
$$

La rapida convergenza delle serie che figurano in questa equazione permette dedurre formule semplificate per il calcolo di $\beta$ applicabili sia nel caso di carico mobile distribuito che concentrato [12], [13], [14].

Per stabilire la relazione che intercorre tra questa equazione e la (52) trascuriamo l'ultimo termine del secondo membro che tiene conto di un effetto non considerato dalla stessa (52), cioè la variazione, lungo il vano, della frazione di carico mobile trasmesso ai tiranti di sospensione.

Per la (89) possiamo scrivere la (92) come segne:

$$
\begin{aligned}
& \sum_{n} 2 \frac{w_{n}}{w_{0}}\left(\frac{l_{n}}{l}\right)^{2} F(z) d \sum_{l}^{z} \sum_{m=1}^{\infty} \frac{(1-\cos m \pi) \operatorname{sen} m \pi Z}{m^{3} \pi^{3} c_{n m}}- \\
& -\beta \sum_{n} 2\left(\frac{w_{n}}{w_{0}}\right)^{2}\left(\frac{l_{n}}{l}\right)^{3} \sum_{m=1}^{\infty} \frac{(1-\cos m \pi)^{2}}{m^{4} \pi^{4} c_{n m}}=\frac{A(1+\beta)}{u^{2}}
\end{aligned}
$$

dove $A$ è definito dalla (50). D'altra parte dalla (84) abbiamo:

$$
\sum_{n} J_{a} F(z) d \frac{z}{l}-\beta \Delta=\frac{A(1+\beta)}{u^{2}}
$$

donde, confrontando con la (52) risulta :

$$
\Delta=G
$$

ciò̀ a dire

$$
G=\sum_{n}\left(\frac{w_{n}}{w_{0}}\right)^{2}\left(\frac{l_{n}}{l}\right)^{3} 2 \sum_{m=1}^{\infty} \frac{(1-\cos m \pi)^{2}}{m^{4} \pi^{4} c_{n m}}
$$

e ancora, per le (57) e (54)

$$
g_{n}=2 \sum_{m=1}^{\infty} \frac{(1-\cos m \pi)^{2}}{m^{4} \pi^{4} c_{n m}} .
$$

Abbiamo così ottenuto le espressioni dedotte precedentemente, sviluppate in serie trigonometriche. 


\section{Funzioni d'influenza.}

Il valore $d \mathrm{i} \eta / l$ in una sezione $X$ della trave irrigidente per un carico distribuito lungo i vani del ponte è dato dalla (44) che possiamo scrivere:

$$
\left.\frac{\eta}{l}=\frac{u}{1+\beta} \mid \int_{T} J_{x z} F(z) d \frac{z}{l}-\beta J_{x}\right]
$$

dove $\int_{T}$ indica che l'integrale è esteso a tutte le travi irrigidenti.

In realtà l'integrale si estende soltanto al vano in eui si trova la sezione $X$ perchè, per gli altri vani, la funzione $J_{x z}$ è nulla.

Osserviamo ohe per la (63) possiamo sorivere:

$$
\beta=\int_{T} \frac{J_{z}}{G} F(z) d \frac{z}{l}-\delta
$$

e sostituendo nella (97):

$$
\frac{\eta}{l}=\frac{u}{1+\beta}\left[\iint_{i}\left(J_{x z}-J_{x} \frac{J_{z}}{G}\right) F(z) d \frac{z}{l}+\delta J_{x}\right] .
$$

In questa equazione, contrariamente a quello che succede con $J_{x z}$, la funzione $J_{x} J_{z} / G$ è sempre diversa da zero, qualunque sia il vano in cui agisea il carico.

L'equazione (99) ha una importanza particolare perchè da essa si deducono facilmente le formule che rendono possibile lo studio completo della trave irrigidente.

Per semplificare le espressioni che dedurremo in seguito, definiamo le seguenti espressioni :

$$
\left\{\begin{array}{l}
J_{w}=\frac{w_{n}}{w_{0}}\left(\frac{l_{n}}{l}\right)^{2} j_{x} \\
J_{x}^{\mathrm{I}}=\frac{l}{l_{n}} \frac{d J_{x}}{d X}=\frac{w_{n}}{w_{0}} \frac{l_{n}}{l} j_{x}^{\mathrm{I}} \\
J_{x}^{\mathrm{II}}=\frac{l_{n}}{c_{n}^{2} l} \frac{d J_{x}^{\mathrm{x}}}{d X}=\frac{w_{n}}{w_{0}}\left(\frac{l_{n}}{l}\right)^{2} j^{\mathrm{II}} \\
J_{x z}^{\mathrm{rIx}}=\frac{l}{l_{n}} \frac{d J_{x}^{\mathrm{II}}}{d X}=\frac{w_{n}}{w_{0}} \frac{l_{n}}{l} j_{x}^{\mathrm{IIx}} \\
J_{x}^{\mathrm{Vx}}=-\frac{l}{l_{n}} \frac{d J_{x}^{\mathrm{III}}}{d X}=\frac{w_{n}}{w_{0}} j_{x}^{\mathrm{IV}}
\end{array}\right.
$$


dove:

$$
j_{x}^{\mathrm{I}}=\frac{d j_{x}}{d X}, \quad j_{x}^{\mathrm{iI}}=-\frac{1}{c_{n}^{2}} \frac{d j_{x}^{\mathrm{I}}}{d X}, \quad j_{x}^{\mathrm{III}}=\frac{d j_{x}^{\mathrm{II}}}{d X}, \quad j_{x}^{\mathrm{iV}}=-\frac{d j_{x}^{\mathrm{III}}}{d \bar{X}}
$$

Poniamo ancora :

$$
\left\{\begin{array}{l}
J_{x z}=\frac{l_{n}}{l} j_{x z} \\
J_{x z}^{\mathrm{x}}=\frac{l}{l_{n}} \frac{\partial J_{x z}}{\partial X}=j_{x z}^{\mathrm{I}} \\
J_{x z}^{\mathrm{II}}=-\frac{1}{c_{n}^{2}} \frac{l_{n}}{l} \frac{\partial J_{x z}^{\mathrm{I}}}{\partial X}=\frac{l_{n}}{l} j_{x z}^{\mathrm{II}} \\
J_{x z}^{\mathrm{III}}=\frac{l}{l_{n}} \frac{\partial J_{x z}^{\mathrm{II}}}{\partial X}=j_{x z}^{\mathrm{IIx}} \\
J_{x z}^{\mathrm{IV}}=-\frac{l}{l_{n}} \frac{\partial J_{x z}^{\mathrm{IIx}}}{\partial X}=\frac{l}{l_{n}} j_{x z}^{\mathrm{IV}}
\end{array}\right.
$$

dove:

$$
j_{x z}^{\mathrm{I}}=\frac{\partial j_{x z}}{\partial X}, \quad j_{x z}^{\mathrm{II}}=-\frac{1}{c_{n}^{z}} \frac{\partial j_{x z}^{\mathrm{I}}}{\partial X}, \quad j_{x z}^{\mathrm{nII}}=\frac{\partial j_{x z}^{\mathrm{II}}}{\partial X}, \quad j_{x z}^{\mathrm{Iv}}=-\frac{\partial j_{x z}^{\mathrm{II}}}{\partial X}
$$

Le funzioni $J_{x z}$ e $J_{\infty}$ sono state già definite dalle (40), (41), (38), (39). Derivando successivamente in rapporto a $X$ l'equazione (99) e applicando le relazioni fondamentali della teoria delle travi rettilinee, otteniamo successivamente per la sezione $X$, la rotazione:

$$
\eta^{\prime}=\frac{d \eta}{d x}=\frac{l}{l_{n}} \frac{d \frac{\eta}{l}}{d X}=\frac{u}{1+\beta}\left[\int_{T}\left(J_{x z}^{\mathrm{x}}-J_{x}^{\mathrm{x}} \frac{J_{z}}{G}\right) F(z) d \frac{z}{l}+\delta J_{x}^{\mathrm{1}}\right]
$$

il momento flettente relativo:

$$
\begin{aligned}
\frac{M}{w_{v} l^{2}}= & -\frac{E J \eta^{\prime \prime}}{u H_{v} l}=-\frac{(1+\beta) E J}{(1+\beta) u H_{v v} l^{2}} \frac{d \eta^{\prime}}{d \frac{x}{l}}=-\frac{(1+\beta) l_{n}^{2}}{\frac{H_{w}(1+\beta)}{E J} u l^{2}} \frac{d \eta_{n}^{2}}{l_{n}^{2}}= \\
& =-\frac{(1+\beta)}{c_{n}^{2} u} \frac{l_{n}}{l} \frac{d \eta^{\prime}}{d X}=\int_{T}\left(J_{x z z}^{1 \mathrm{n}}-J_{x}^{\mathrm{II}} \frac{J_{z}}{G}\right) F(z) d \frac{z}{l}+\delta J_{x}^{\mathrm{II}}
\end{aligned}
$$

lo sforzo tagliante relativo:

$$
\frac{Q}{w_{0} l}=\frac{1}{w_{0} l^{2}} \frac{d M}{d \frac{x}{l}}=\frac{l}{l_{n}} \frac{d}{d X}\left(\frac{M}{w_{0} l^{\bar{z}}}\right)=\int_{T}\left(J_{x z}^{\mathrm{III}}-J_{x z}^{\mathrm{III}} \frac{J_{z}}{G}\right) F(z) d \frac{z}{l}+\delta J_{x}^{\mathrm{III}}
$$


la frazione relativa di carico mobile che agisce sulla trave irrigidente:

$$
\frac{p_{T}}{w_{0}}=-\frac{1}{w_{0} l} \frac{d Q}{d \frac{x}{l}}=-\frac{l}{l_{n}} \frac{d}{d X}\left(\frac{Q}{w_{\mathrm{l}} l}\right)=\int_{T}\left(J_{x z}^{\mathrm{iv}}-J_{x^{\prime}}^{\mathrm{rv}} \frac{J_{z}}{G}\right) F(z) d \frac{z}{l}+\delta J_{x}^{\mathrm{IV}}
$$

dove :

$$
p_{T}=p-p_{t}
$$

essendo $p_{t}$ la frazione di carico mobile trasmessa ai tiranti di sospensione.

Se poniamo:

$$
I_{x z}^{(i)}=J_{x z}^{(i)}-, I_{x z}^{(i)} \frac{J_{z}}{G}
$$

abbiamo:

$$
\begin{gathered}
\left.\frac{\eta}{l}=\frac{u}{1+\beta} \mid \int_{T} I_{x z} F(z) d \frac{z}{l}+\delta J_{x}\right] \\
\left.\eta^{\prime}=\frac{u}{1+\beta} \mid \int_{T} I_{x z}^{\mathrm{I}} F(z) d \frac{z}{l}+\delta J_{x}^{\mathrm{I}}\right] \\
\frac{M}{w_{0} l^{2}}=\int_{T} I_{x z}^{\mathrm{II}} F(z) d \frac{z}{l}+\delta J_{x}^{\mathrm{II}} \\
\frac{Q}{w^{\prime \prime} l}=\int_{T} I_{x z}^{\mathrm{III}} F(z) d \frac{z}{\bar{l}}+\delta J_{x}^{\mathrm{III}} \\
\frac{p_{T}}{w_{0}}=\int_{T} I_{x z}^{1 \mathrm{v}} F(z) d_{\bar{l}}^{z}+\delta J_{x z}^{\mathrm{Iv}} .
\end{gathered}
$$

Osserviamo che $p_{T}=p-p_{t}$. Se nella sezione $X$ non agisce nessun carico $p$ risulta $p_{T}=-p_{t}$, cioè la trave irrigidente, nella sezione in esame, è soggetta solamente all'azione dei tiranti di sospensione. È necessario osservare che la funzione $I_{x: z}^{11 x}$ dello sforzo di taglio soffre nella sezione $X$ un salto. I valori delle derivate, però, $d I_{x z}^{\mathrm{III}} / d X=\ldots I_{x z}^{\mathrm{IV}}$, subito a destra e a sinistra dellat sezione $X$ sono uguali, per cui se si trascura il salto della funzione $I_{x z}^{111}$ nel calcolo di $I_{x z}^{\mathrm{lv}}$, si traseura anche l'influenza del carico $p$ che agisce nella sezione $X$. In questo caso il secondo membro della (113) fornisce in realtà, il valore $\mathrm{di}-p_{t} / w_{0}$ nella sezione $X$.

Con le (109), (110), (111), (112) e (113) possiamo calcolare per una sezione $X$ della trave irrigidente, in un vano qualunque del ponte, $\mathrm{i}$ valori $\mathrm{di} \eta, \eta^{\prime}, M$, $Q, p_{t}$ per una condizione di carico assegnata, nell'ipotesi di $c_{n}$ costante. Da questa osservazione risulta che trascurando le correzioni, Ie funzioni $I_{x z}^{(i)}$ sono funzioni d'influenza ristretta, ciò̀ per $c_{n}$ costante. 
Conoscinte, pertanto, le funzioni $I_{x z}^{(i)}$ d'influenza e supposto $\delta=0$, pos. siamo tracciare le linee d'influenza e determinare le condizioni più sfavorevoli di carico per $\mathrm{i}$ diversi effetti in una sezione $X$, ammesso $c_{n}=$ costante. Le linee d'influenza sarebbero esatte se $\delta$ fosse realmente nullo e se le travi irrigidenti variassero automaticamente la caratteristica $E J_{n}$ al variare la spinta totale, di maniera a soddisfare la condizione $c_{n}=$ costante, il che non si realizza in pratica. I calcoli, pertanto, dovranno essere condotti col metodo delle approssimazioni successive. Si osservi che $c_{n}$ dipende dalla condizione più sfavorevole di carico per l'effetto in osame.

D'altra parte la condizione più sfavorevole di carico, determinata dalla relativa linea d'influenza, dipende da $c_{n}$. E necessario, pertanto, ammettere in prima approssimazione, un conveniente valore di $c_{n}$ e tracciare la linea d'influenza di $\beta_{0}$ e dell' effetto ohe si desidera calcolare. Saranno conosciute, pertanto, in prima approssimazione, le sezioni tra le quali è necessario distribuire il carico. La linea d'influenza di $\beta_{0}$ fornirà, allora, il valore di $\beta_{0}$ rela. tivo a questa condizione di carico. Si corregge in seguito $\beta_{0}$ tenendo conto del termine correttivo che dipende anche esso da $c_{n}$.

Si ottiene cosi $\beta^{\prime}$ e pertanto un valore più esatto di $c_{n}$. Con questo nuovo valore di $c_{n}$ si tracciano nuovamente le linee d'influenza di $\beta_{0}$ e dell' effetto che si desidera calcolare; si ripete cosi il calcolo $\theta$ si ottiene un nuovo valore $\beta^{\prime \prime}$, e cosi di seguito. $\mathbf{E}$ importante osservare ohe la convergenza del processo è molto rapida. Tracciata la linea d'influenza definitiva si calcola il valore massimo dell' effetto in esame, tenendo conto del termine correttivo già al calcolare l'ultimo valore $\beta(i)$. Il lavoro di calcolo viene di molto ridotto se si adopera un processo d'interpolazione [10] tra casi opportunamente scelti.

Osserviamo che possiamo scrivere la (108) come segue:

$$
I_{x z}^{(i)}=J_{x z}^{(i)}-J_{x}^{(i)} \frac{J_{z}}{G_{z}} \frac{G_{z}}{G} .
$$

Si noti che quando il carico mobile agisce in una sezione $Z$ di un vano diverso da quello al quale appartiene la sezione $X$ che si considera, il termine $J_{x z}^{(i)}$ si annulla.

Sostituiamo nella (114) in luogo di $J_{x z}^{\{i}$ e $J_{a}^{\{i\}}$ i valori corrispondenti forniti dalle (102) e (100), e in luogo di $J_{z} / G_{z}$ il valore fornito dalla (66).

Otteniamo, pertanto, per le diverse funzioni d'influenza le seguenti espressioni :

$$
\begin{aligned}
& I_{x z}=\left(j_{x z}-\frac{w_{x}}{w_{z}} \frac{l_{x}}{l_{z}} \frac{G_{z}}{G} j_{x} \frac{j_{z}}{g_{z}}\right) \frac{l_{x}}{l} \\
& I_{x z}^{\mathrm{I}}=j_{x z}^{\mathrm{I}}-\frac{w_{x}}{w_{z}} \frac{l_{x}}{l_{z}} \frac{G_{z}}{G} j_{x}^{\mathrm{I}} \frac{j_{z}}{g_{z}}
\end{aligned}
$$




$$
\begin{aligned}
& I_{x z}^{\mathrm{II}}=\left(j_{x z}^{\mathrm{II}}-\frac{w_{x}}{w_{z}} \frac{l_{x}}{l_{z}} \frac{G_{z}}{G} j_{x}^{\mathrm{ir}} \frac{j_{z}}{g_{z}}\right) \frac{l_{x}}{l} \\
& I_{x z z}^{\mathrm{II}}=j_{x z}^{\mathrm{III}}-\frac{w_{x}}{w_{z}} \frac{l_{x}}{l_{z}} \frac{G_{z}}{G} j_{x}^{\mathrm{mI}} \frac{j_{z}}{g_{z}} \\
& I_{x z}^{\mathrm{IV}}=\left(j_{x z}^{\mathrm{IV}}-\frac{w_{x}}{w_{z}} \frac{l_{x}}{l_{z}} \frac{G_{z}}{G} j_{x}^{\mathrm{IV}} \frac{j_{z}}{g_{z}}\right) \frac{l}{l_{x}} .
\end{aligned}
$$

Gli indici $x$ e $z$ si riferiscono ai vani a cui appartengono, rispettivamente, la sezione $X$ in esame e la sezione $Z$ nella quale agisce il carico. Le espressioni delle funzioni $j_{x}^{(i)}$ e $j_{x z}^{(i)}$ si deducono facilmente. Conosciamo, difatti, le espressioni di $j_{x}$ e di $j_{x z}$ fornite dalle (39) e (38). Derivando successivamente in rapporto a $X$ e tenendo conto della definizione delle diverse funzioni fornite dalle (101) abbiamo:

$$
\left\{\begin{array}{l}
j_{x}=\frac{1}{2} X(1-X)-\frac{1}{c_{n}^{2}}+\frac{\operatorname{ch} c_{n}\left(\frac{1}{2}-X\right)}{c_{n}^{2} \operatorname{ch} \frac{c_{n}}{2}} \\
j_{x}^{\mathrm{t}}=\frac{1}{2}-X-\frac{\operatorname{sh} c_{n}\left(\frac{1}{2}-X\right)}{c_{n} \operatorname{ch} \frac{c_{n}}{2}} \\
j_{x}^{\mathrm{II}}=\frac{1}{c_{n}^{2}-\frac{\operatorname{ch} c_{n}\left(\frac{1}{2}-X\right)}{c_{n}^{2} \operatorname{ch} \frac{c_{n}}{2}}} \\
j_{x}^{\mathrm{nI}}=\frac{\operatorname{sh~} c_{n}\left(\frac{1}{2}-X\right)}{c_{n} \operatorname{ch} \frac{c_{n}}{2}} \\
j_{x}^{\mathrm{IV}}=\frac{\operatorname{ch~} c_{n}\left(\frac{1}{2}-X\right)}{\operatorname{ch} \frac{c_{n}}{2}} .
\end{array}\right.
$$

Si osservi che in relazione alla sezione di mezzo del vano le funzioni $j_{x}, j_{x}^{\text {II }}$ e $j_{x}^{1 \mathrm{~V}}$ sono simmetriche, mentre le $j_{x}^{\mathrm{I}}, j_{x}^{\mathrm{III}}$ sono antisimmetriche. 
Dalle (103) risulta per le funzioni $j_{x z}^{(i)}$ :

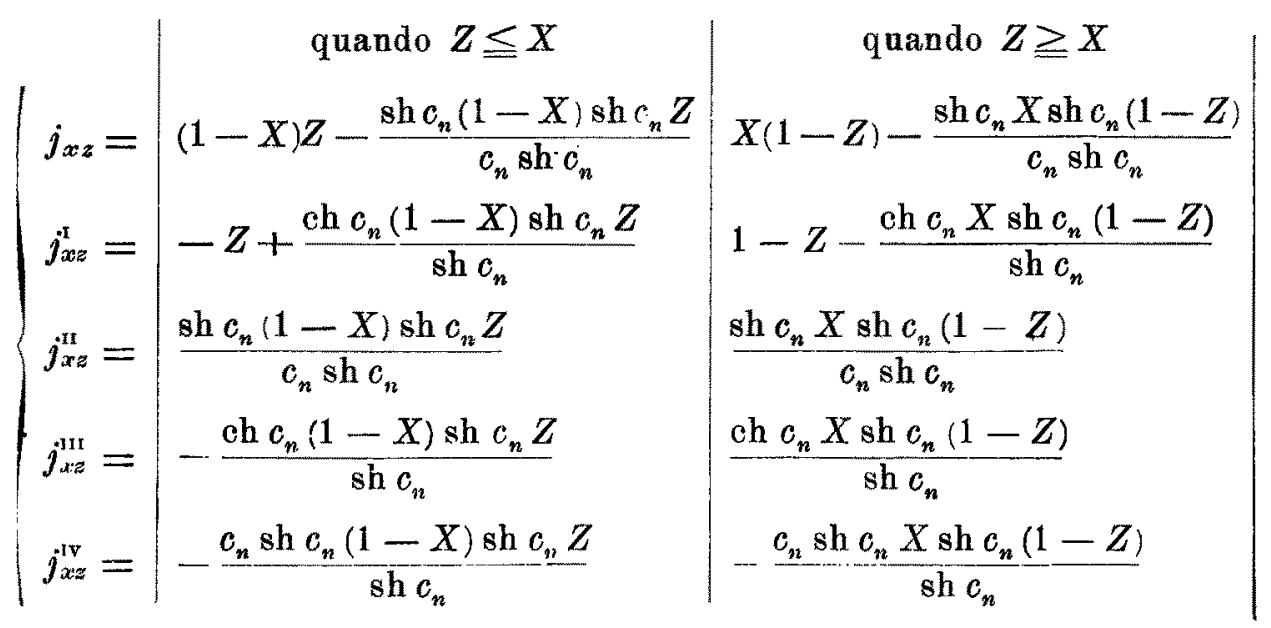

Si osservi che in relazione alla sezione di mezzo del vano, cio®̀ $X=\frac{1}{2}$, le funzioni $j_{x z}, j_{x z}^{\mathrm{II}}$ e $j_{x z}^{1 \mathrm{vV}}$ sono simmetriche mentre $j_{x z}^{\mathrm{I}}$ e $j_{x z}^{\mathrm{IIr}}$ sono antisimmetriche.

Casi particolari delle formule innanzi dedotte sono quelle relative alla Teoria Elastica, come abbiamo già osservato. Otteniamo, pertanto, passando al limite $c_{n}=0$ :

$$
\left\{\begin{array}{l}
j_{x 0}=\frac{c_{n}^{2}}{24} X(1-X)\left(1+X-X^{2}\right) \\
j_{x 0}^{\mathrm{I}}=\frac{c_{n}^{2}}{24}(1-2 X)\left(1+2 X-2 X^{2}\right) \\
j_{x 0}^{\mathrm{II}}=\frac{1}{2} X(1-X) \\
j_{x 0}^{\mathrm{IIX}}=\frac{1}{2}-X \\
j_{x 0}^{\mathrm{IV}}=1
\end{array}\right.
$$

e ancora:

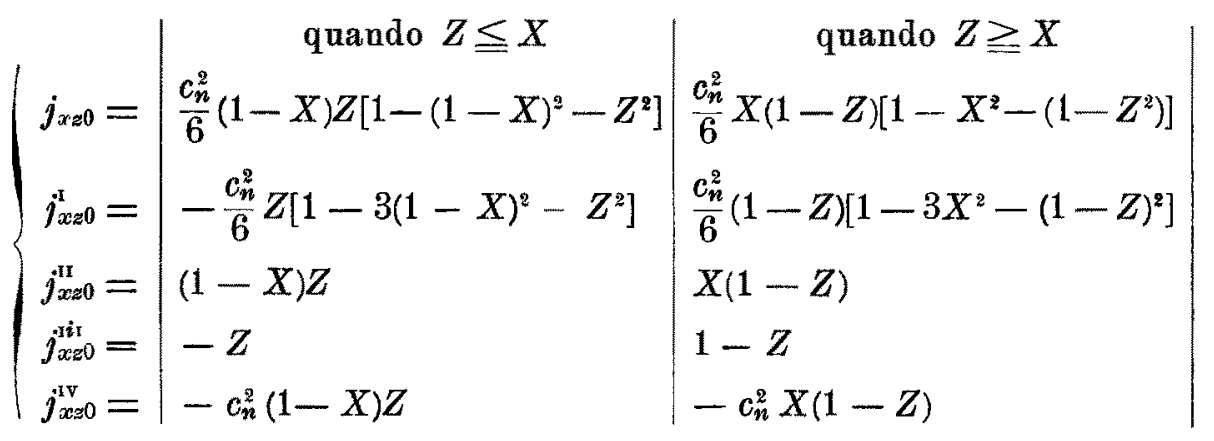




\section{Otteniamo inoltre:}

$$
g_{n 0}=\frac{c_{n}^{2}}{120}
$$

Se passiamo al limite per $c_{n}=\infty$ deduciamo le formule relative al cavo isolato :

$$
\left\{\begin{array}{l}
j_{x \infty 0}=\frac{1}{2} X(1-X) \\
j_{x \infty o}^{\mathrm{I}}=\frac{1}{2}-X \\
j_{x \infty}^{\mathrm{II}}=\frac{1}{c_{n}^{2}} \\
j_{x \infty 0}^{\mathrm{IIx}}=\frac{e^{-c_{n} X}}{c_{n}} \\
j_{x \infty 0}^{\mathrm{IV}}=e^{-c_{n} X}
\end{array}\right.
$$

e ancora:

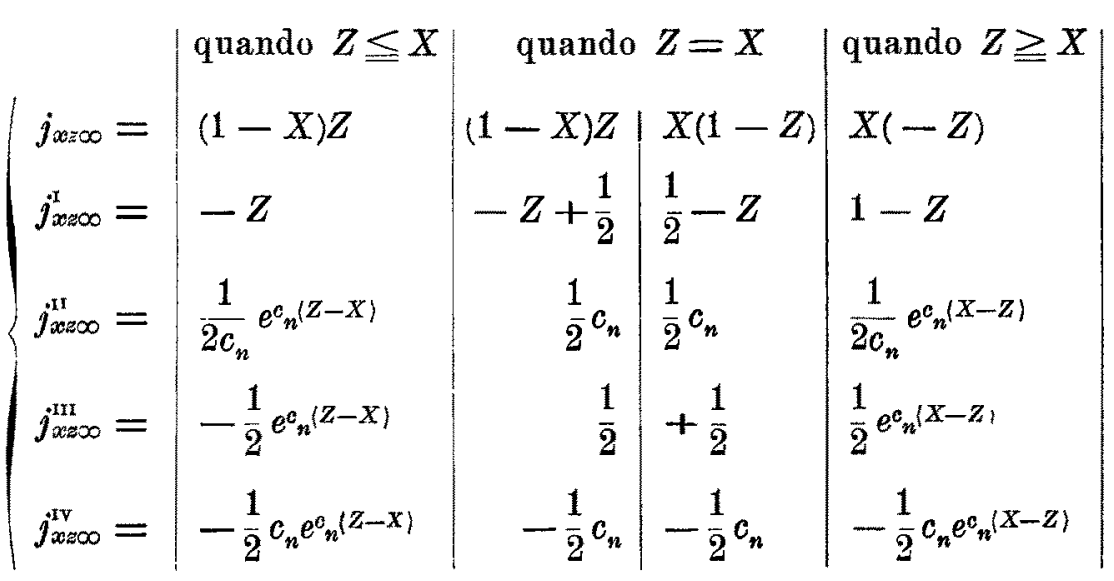

Otteniamo inoltre:

$$
g_{n \infty}=\frac{1}{12}
$$

E facile dedurre le espressioni di $j_{x}^{(i)}$ e $j_{x z}^{(i)}$ sviluppate in serie trigonometriche. Difatti conosciamo le espressioni (86) e (85) di $j_{x}$ e $j_{x z}$. 
Derivando successivamente in rapporto a $X$ e tenendo conto della definizione delle diverse funzioni date dalle (101) e (103) deduciamo:

$$
\left\{\begin{array}{l}
j_{x}=2 \sum_{m=1}^{\infty} \frac{(1-\cos m \pi) \operatorname{sen} m \pi X}{m^{3} \pi^{3} c_{m m}} \\
j_{x}^{\mathrm{I}}=2 \sum_{m=1}^{\infty} \frac{(1-\cos m \pi) \cos m \pi X}{m^{2} \pi^{2} c_{n m}} \\
j_{x}^{\mathrm{II}}=\frac{2}{c_{n}^{2}} \sum_{m=1}^{\infty} \frac{(1-\cos m \pi) \operatorname{sen} m \pi X}{m \pi c_{m m}} \\
j_{x}^{\mathrm{III}}=\frac{2}{c_{n}^{2}} \sum_{m=1}^{\infty} \frac{(1-\cos m \pi) \cos m \pi X}{c_{n m}} \\
j_{x}^{\mathrm{IV}}=\frac{2}{c_{n}^{2}} \sum_{m=1}^{\infty} \frac{m \pi(1-\cos m \pi) \operatorname{sen} m \pi X}{c_{n m}} .
\end{array}\right.
$$

E ancora:

$$
\left\{\begin{array}{l}
j_{x}=2 \sum_{m=1}^{\infty} \frac{\operatorname{sen} m \pi Z \operatorname{sen} m \pi X}{m^{2} \pi^{2} c_{m n}} \\
j_{x z}^{\mathrm{I}}=2 \sum_{m=1}^{\infty} \frac{\operatorname{sen} m \pi Z \cos m \pi X}{m \pi c_{n m}} \\
j_{x z}^{\mathrm{II}}=\frac{2}{c_{n}^{2}} \sum_{m=1}^{\infty} \frac{\operatorname{sen} m \pi Z \operatorname{sen} m \pi X}{c_{n m}} \\
j_{x z}^{\mathrm{III}}=\frac{2}{c_{n}^{2}} \sum_{m=1}^{\infty} \frac{m \pi \operatorname{sen} m \pi Z \cos m \pi X}{c_{n m}} \\
j_{x z}^{\mathrm{IV}}=\frac{2}{c_{n}^{2}} \sum_{m=1}^{\infty} \frac{m^{2} \pi^{2} \operatorname{sen} m \pi Z \operatorname{sen} m \pi X}{c_{n m}}
\end{array}\right.
$$

Otteniamo inoltre:

$$
g_{n}=2 \sum_{m=1}^{\infty} \frac{(1-\cos m \pi)^{2}}{m^{4} \pi^{4} c_{n m}}
$$

Per semplificare l'applicazione delle formule dedotte in questa memoria e il calcolo che si propone per i ponti sospesi mediante le funzioni di influenza, abbiamo determinato [15] per diversi valori di $c_{n}, X_{n}$ e $Z_{n}$, i valori delle funzioni $j_{x}^{\langle i\rangle}, j_{i x z}^{i i\rangle}$ e $g_{n}$. I risultati, notevolmente ampliati con la considerazione di numerosi valori delle variabili, saranno pubblicati prossimamente a cura dell' Istituto Nazionale per le Applicazioni del Calcolo. 
Riteniamo che in tal maniera il calcolo mediante la teoria del secondo ordine dei ponti sospesi, soggetti all'azione di carichi, considerati agenti staticamente, non offrirà più difficoltà degne di nota, riducendosi a una serie di operazioni elementari.

\section{BIBLIOGRAFIA}

[1] Navier L., Mémoire sur les ponts suspendus, Paris, 1823.

[2] MüLler Bresla U H. F. B., Theorie der aurch einen Balken versteiften Kette, "Zeitsohrift des Arkitekten- und Ingenieur. Vereins zu Hannover s, 1881.

[3] CaDaRT G., Théorie des ponts suspendus américains à tablier rigide, Annales des Ponts et Chaussees, 1885.

[4] Levy M., Mémoire sur le calcul des ponts suspendus rigides, Annales des Ponts et Chaussées », 1886.

[5] Melan J., Handbuch der Ingeniewrwissenschaften, Leipzig, 1888.

[6] Martin, The theory of the stiffened suspension bridge, "Engineering ", 1927.

[7] Trmoshenko S., The stiffness of suspension bridges, * A. S. C. E. », 1928.

[8] STussi F., Zuv Berechnung verankerter Hängebrücken, "A. I. P. C. ", 1936.

[9] Rudbero H., Matematisk behanalling av hängbroar, "Tekniska Skrifter », 1944.

[10] Aspuund S. O., On the deflection theory of suspension bridges, Stockholm, 1945.

[11] Selberg A, Design of suspension bridges, Trondheim, 1946.

[12] Gravina P. B. J., Teoria das pontes pênseis. São Paulo, 1948.

[13] Gravina P. B. J., Nota sôbre o cálculo do empuxo adicional nos cabos das pontes pênseis, angenharia », 1949.

[14] Gravina P. B. J., Contribuição para o calculo do empuxo adicional nos cabos das pontes pênseis. Sobre uma equação simplificada aplicável no caso de carga movel distribuida e sua generalização para qualquer condição de carga, « D. E. R. », São Paulo, 1949.

[15] Gravina P. B. J., Teoria e cálculo das pontes pềnzeis, São Paulo, 1951. 